\title{
Research on the Application of Computer Information Technology in Network Security under the Background of "Big Data"
}

\author{
Liu Ying \\ Xijing University, Xi`an Shaanxi 710123, China
}

Keywords: "big data"; computer information technology; network security; application

\begin{abstract}
At present, computer information technology is highly valued by all walks of life. To this end, it is necessary to ensure that people have a good understanding of computer information technology. In addition, under the background of "big data", there are still some security problems in the long-term operation of network systems in various industries in China, which affect the application value of computer network systems. This paper will give a comprehensive overview of the implementation of network security under the background of "big data", and optimize the network security issues with the support of computer information technology, so as to provide dynamic support for the sound development of various industries in China.
\end{abstract}

\section{Introduction}

As an important development direction in the era of "big data", the application of computer information technology can improve network security to a certain extent, highlight the level of network security under the background of "big data", and implement the goal of expanding the application scope of network technology. From the point of view of network security, we know that the network security problem under the background of "big data" is more complex, which will increase the difficulty of dealing with the corresponding problems invisibly, and affect the development effect of "big data" era in our country. Based on this, we should effectively deal with the network security problems with the support of computer information technology to ensure the wide application of computer information technology under the background of "big data".

\section{Overview of Computer Information Technology}

Within a long period of time, computer information technology has developed rapidly. This development direction can not only expand the relevant personnel's grasp of computer information technology, but also solve a series of network security problems with the help of computer information technology. Continuous optimization of the development trend of computer information technology under the background of "big data" has widely expanded the application scope of computer information technology. From the point of view of computer information technology, we know that computer information technology is mainly manifested in the use of computer equipment to study various information. Through a series of studies, it can strengthen people's grasp of various information to a certain extent, and adjust unreasonable working methods accordingly. Comparing with traditional information technology, computer information technology can achieve the goal of improving the level of information storage to a certain extent, and reduce the time and energy invested in the research and development and utilization of information. Continuous optimization of the outcome effect of various information has already laid a solid foundation for the steady development of China's "big data" era. Moreover, with the support of computer information technology, it can better solve a series of network security problems arising from the development of "big data" era, reduce the impact of network security issues on the development of the "big data" era, and highlight the charm of the development of the information age. 


\section{Causes of Network Security Problems under the Background of "Big Data"}

Although the development of the "big data" era can improve the traditional network operation mode, it will also lead to a series of security problems in the network system, which directly affects the network security effect under the background of "big data", and also has a great impact on the basic work of the internet of various industries. Based on this, we should strengthen the research on network security issues under the background of "big data", understand the causes of network security problems, and plan reasonable solutions for the later period. At present, there are many incentives for network security problems under the background of "big data", mainly in the following aspects:

\subsection{Low Openness of Internet}

Influenced by the "big data" background, the effect of Internet opening has been greatly improved, which requires relevant personnel to carry out an effective analysis of the operation mode of the Internet to improve the development interests of various industries in China, while expanding the scope of Internet applications. However, under such conditions, a large number of lawbreakers accordingly use the Internet model to carry out various fraudulent activities. Not only will it lead to the decline of the level of network security, but also will lead to the fraud of lawbreakers in various industries in our country. It will also have a great impact on the comprehensive development benefits of various industries. In addition, the low level of comprehensive maintenance of computer network systems applied in various industries and the not complete development to the mature level of the self-protection of network systems have affected the security of information transmission of network systems to increase the possibility of network security problems under the background of "big data", and hinder the development of information age in China.

\subsection{Improper Use of Computer Networks}

When putting computers into various applications, it is necessary not only to ensure that the relevant personnel have a good grasp of the characteristics of computer functions, but also to require the relevant personnel to operate the computer according to the prescribed process, fully demonstrating the application advantages of computer networks, so that the role of computer networks can be fully played. Because of the complexity of computer network system, it is difficult for the relevant personnel to master the operation mode of computer network in a short time, which increases the possibility of problems in the application of computer network and has a great impact on the security of computer network operation. In addition, some computer operators lack sufficient security awareness and cannot solve computer network security problems in accordance with relevant requirements. As a result, under the background of "big data", the problem of network security becomes expanding indefinitely, and the hidden dangers of network security emerge endlessly. If this phenomenon is laissez-faire, it will also lead to the leakage of security passwords and identity information when operators use computer networks in severe cases,, which will bring serious security risks to computer network applications.

\subsection{Invasion by virus}

In the process of long-term operation of computer equipment, there will not only be system damage, but also the low security level of computer network system, and the probability of computer network system being threatened by virus will be greatly increased. Although "big data" can bring some convenience for people to implement various tasks, it will also lead to the increase of the probability of virus intrusion into computer networks, resulting in endless computer network security problems. And in the process of in-depth study of computer network viruses, we come to know that computer network viruses have the characteristics of fast transmission rate and difficult access to query. Once the computer network is affected by the virus, it will inevitably lead to the disorder of the computer network system and the serious loss of all kinds of information, which will also have a great impact on the operation effect of the computer network system. In addition, under 
the condition that computers are connected with foreign equipment, the probability of virus infection becomes greatly increased, which affects the network security under the background of "big data", resulting in endless network security problems.

\subsection{Illegal hacker attacks}

In addition to virus interference, computer networks will also be attacked by hackers, resulting in the loss of useful data and information in computer network systems, and the decline of computer network system operation mode is difficult to meet the application requirements of computer network systems. Moreover, most users are accustomed to storing data information in the computer network. Hackers attack the corresponding network system in order to steal the information they need. It results in the loss and leakage of information stored in computer networks and leads to the decline of network system operation effect under the background of "big data", and causes the emergence of network security problems, which seriously affects the effect of the corresponding network system. If we cannot effectively deal with the problem of illegal hacker attacks, it will inevitably lead to the collapse of computer network system and seriously hinder the development of information age in our country.

\section{Application of Computer Information Technology in Network Security under the Background of "Big Data"}

From the above discussion, we can see that under the background of "big data", computer network security will be disturbed by many external factors, which requires planning network security management measures according to the corresponding analysis to effectively control network security issues, thereby improving network security under the background of "big data". At present, under the background of "big data" when optimizing the network security issues, computer information technology will be applied. Continuous improvement the security problems produced in the operation of computer networks, and the highlight of the security effects of computer networks, lead to the full play of the effect of computer information technology. At present, under the background of "big data", computer information technology has obvious application in network security, which mainly manifests in the following aspects:

\subsection{Enhancement of the awareness of network security protection of relevant personnel}

Because there are essential differences in the security problems arising from the operation of computer networks, it is necessary to ensure that the relevant personnel have sufficient knowledge of the network security issues. At the same time, under the support of computer information technology, the network security problems under the background of "big data" becomes effectively analyzed, and the incentives of security problems of the network under the background of "big data" becomes clearly defined. Accordingly reasonable solutions are planned to implement the goal of improving the level of network security. At the same time, we should organize effective training for computer network security maintenance personnel, improve the relevant personnel's eyesight of network security maintenance, constantly improve the security awareness of computer network users, and then plan reasonable solutions with the support of computer information technology, so as to continuously improve the level of the operation security of computer network and expand the scope of computer network applications. Moreover, there are many security protection modes involved in computer network system, which requires relevant personnel to establish safety management concept in the management of corresponding network system, effectively enhance the application value of computer information technology in network security management, and ensure the smooth development of network security protection work. If a series of network security problems arise under the interference of external factors in the computer network system, the relevant personnel can be required to carry out the optimized management on the network security problems and adjust the hidden problems in the computer network in combination with their own awareness of network security protection. Optimization of the computer network operation procedures has laid a solid foundation for improving the effect of computer network security 
management.

\subsection{Establishment of a Perfect Network Security Management System}

From the point of view of the development of "big data" era, we should strengthen the application value of computer information technology in network security management, timely and effectively solve the security problems of computer network system in the process of long-term operation, and vigorously promote the development of computer network system in China towards a reasonable direction. Because the reasons of network security problems under the background of "big data" are rather complex, we should establish a perfect security management mechanism according to the causes of network security problems, gradually improve the convenience of network security issues, and effectively control the impact of network security issues on the development of "big data" era. For the emergent security problems of computer network system, it is also necessary to improve the emergency response ability of relevant personnel under the support of relevant management mechanism, and to implement the goal of improving the level of network security management from a fundamental perspective. In addition, a series of modern laws and regulations should be applied in computer information technology, and correct guidance and management of computer network security issues should be implemented with the support of various laws and regulations. Relevant personnel's grasp of the moral bottom line of various network security issues should be strengthened to avoid the phenomenon of crossing the boundary in computer network security management, and ensure that network security under the background of "big data" can develop towards a more reasonable direction with the support of computer information technology.

\subsection{Flexible Application of Computer Information Network Firewall Security Technology}

In order to avoid the influence of virus and hacker on computer network security, it is necessary not only to carry out effective analysis on aspects such as network virus and hacker attack ways, but also to create firewall security technology in the process of computer network operation in combination with computer information technology and to improve computer network security level under the control of firewall security technology to avoid the access of lawbreakers into the computer network system. In the process of in-depth analysis of computer network firewall security technology, we understand that the application of this technology can plan different levels of computer network security protection mode, and create a firewall structure between computer network and external environment, so as to ensure the stability of computer information network and fundamentally enhance the security effect of computer network. In addition, computer information technology and network firewall security technology can also be combined to carry out an in-depth analysis of the functional attributes of the network firewall system and various information transmission channels to ensure the stability of information transmission in computer network system and effectively control the impact of dangerous plug-ins on computer network security. For the rather complex computer network system, we should also ensure that the computer network and other network security products are in a state of integration, and further create a more reasonable network security operation environment for computer users.

\subsection{Implementing Effective Detection of Computer Network System}

With the continuous development of the "big data" era, various computer networks have been used efficiently. Although this can improve the unreasonable phenomena in the process of computer network application, it will also lead to more and more kinds of computer network viruses, which poses a great threat to computer network security. In order to improve this situation, we need to develop a series of computer network system detection technologies based on computer information technology to control the impact of various network viruses on computer network security in the process of effective detection of computer network system. At present, there are many detection technologies involved in computer information network system, including signature analysis technology and statistical analysis technology. Therefore, effective analysis of these two network system detection technologies should be carried out, and effective analysis of computer network 
system security issues with the support of various technologies should be carried out. The security risk of gap network system should be further inferred, and corresponding measures to solve the corresponding problems should be formulated to maximize the protection of computer network security and highlight the application value of computer information technology in network security.

\section{Conclusion}

Above all, the understanding of the development of the "big data" era can not only improve the development pattern of the information age in our country, but also play a very important role in improving the application effect of the "big data" model. However, under the background of "big data", a series of security problems will appear in various network systems, which will directly affect the effect of various network systems, resulting in stagnation of the development of "big data" era. Therefore, we should optimize and update computer information technology under the background of "big data", and deal with various network security problems with the support of computer information technology, so as to enhance the operation security of various network systems.

\section{References}

[1] Xiong Xiaodong. Reflections on the development trend of network security and information technology [J]. Digital World. 2018 (01).

[2] He Zhiheng. Research on Network Security and Information Technology Development Trend [J]. Digital Communication World. 2017 (10).

[3] Peng Shichun. Design of effective application mode of computer information technology based on network security analysis [J]. Journal of Heihe University. 2017 (10).

[4] Gong Zhiyuan. The Application of Computer Information Technology in Network Security under the Background of "Big Data" [J]. Farmer's Staff. 2018 (09).

[5] Chen Zhengyi. Brief analysis of network security in the process of rapid development of information technology [J]. Network security technology and application. 2018 (03). 